\title{
A proposal to minimize the dispersion on primary calibration results of single-ended accelerometers at high frequencies
}

\author{
Gustavo P. Ripper, Giancarlo B. Micheli, Ronaldo S. Dias \\ INMETRO-Instituto Nacional de Metrologia, Qualidade e Tecnologia, Av. N.S. das Graças 50, Xerém, D. Caxias, 25250-020 R.J., Brazil
}

ABSTRACT

Primary calibrations of single-ended accelerometers by laser interferometric methods can be strongly affected at high frequencies by the vibration exciter used to generate the mechanical motion. A study was carried out at the Vibration Laboratory of INMETRO aiming to better understand the causes of this dependence and to find a way to reduce its effect. A simple solution is proposed by the authors to reduce the dispersion on the sensitivity results evidenced when different vibration exciters are used. This proposal can be very helpful for calibration laboratories willing to compare results obtained in different measurement systems associated with low measurement uncertainties. Therefore, it might benefit processes, which includes for example the validation of calibration systems and interlaboratory comparisons.

\section{Section: RESEARCH PAPER}

Keywords: Primary calibration; vibration; accelerometer; metrology; interferometer

Citation: Gustavo P. Ripper, Giancarlo B. Micheli, Ronaldo S. Dias, A proposal to minimize the dispersion on primary calibration results of single-ended accelerometers at high frequencies, Acta IMEKO, vol. 2, no. 2, article 9, December 2013, identifier: IMEKO-ACTA-02 (2013)-02-09

Editor: Paolo Carbone, University of Perugia

Received February $20^{\text {th }}, 2013$; In final form April 27 ${ }^{\text {th }}, 2013$; Published December 2013

Copyright: (C) 2013 IMEKO. This is an open-access article distributed under the terms of the Creative Commons Attribution 3.0 License, which permits unrestricted use, distribution, and reproduction in any medium, provided the original author and source are credited

Funding: This work was supported by the National Institute of Metrology, Quality and Technology - INMETRO, Brazil

Corresponding author: Gustavo P. Ripper, e-mail: gpripper@inmetro.gov.br

\section{INTRODUCTION}

Primary interferometric calibrations of accelerometers are usually performed at National Metrology Institute (NMI) level in accordance with the international standard ISO 16063-11 [1]. The determination of the sensitivity of an accelerometer is based on the measurement of its electrical output while a given acceleration input is measured at its reference surface. In the case of single-ended accelerometers (SE), the interferometric measurement of the input motion quantity is usually made on the moving table of the vibration exciter, close to the accelerometer's mounting base, since its reference mounting surface is directly not accessible.

CIPM key comparisons [2] are the best way currently available to establish a reference value and to assess the degree of equivalence between different realizations of primary calibration systems developed by the NMIs worldwide.

For the first CIPM vibration key comparison CCAUV.V$\mathrm{K} 1$, two accelerometers, one back-to-back and one singleended, were circulated for primary calibration of the sensitivity magnitude between $40 \mathrm{~Hz}$ and $5 \mathrm{kHz}$. Unfortunately, no key comparison reference value could be determined above $2 \mathrm{kHz}$ for the SE accelerometer. This was caused by a large dispersion observed on the results reported by the participants at high frequencies [3].

Considering that several NMIs have updated their calibration systems and improved their measurement capabilities, the following CIPM key comparison CCAUV.V$\mathrm{K} 2$ was designed to cover a broader scope of measurement. Its technical protocol [4] requests the calibration of both magnitude and phase shift of the complex sensitivity of two accelerometers between $10 \mathrm{~Hz}$ and $10 \mathrm{kHz}$.

During the preliminary stage used for monitoring the stability of the accelerometers, the pilot laboratory (PTB/Germany) needed to replace its high frequency vibration exciter with another model of different armature material. PTB has then observed considerable differences between the sensitivities determined using their new exciter in comparison to the ones obtained with the prior one. This has been reported by Dr. Thomas Bruns in 2009, during the IMEKO TC 22 technical meeting in Lisbon. Later on, two articles related to this problem were published in Metrologia [5][6]. 
The same behavior has also been observed at the vibration laboratory of INMETRO during the validation stage of our primary quadrature interferometric calibration system, when different vibration exciters were tested. It was realized that this problem could be the major source of error and a limiting factor for the future extension of Calibration and Measurement Capabilities up to $20 \mathrm{kHz}$. The authors have then decided to study this problem closer and carry out a research aiming to reduce the systematic deviation observed at high frequencies.

A proposal to minimize this effect is presented herein, which can certainly benefit other laboratories that want to carry out internal validations through comparisons between their own different calibration systems. It can also be helpful to future interlaboratory key and supplementary comparisons in vibrations.

In the next section 2 we will present the level of dispersion normally observed in calibrations at high frequencies. In section 3 and 4 the mathematical model of an ideally mounted SE accelerometer is presented and the effect of the mounting surface quality is discussed. The use of a mechanical adapter with high stiffness is proposed in section 5 and it is illustrated how it can help to maintain a fixed reference measuring condition in section 6 . Some experimental results are presented in section 7 to demonstrate the level of improvement that can be achieved and in sequence, conclusions are summarized.

\section{DISPERSION OBSERVED IN DIFFERENT EXCITERS}

Bruns et al. [5] have reported the comparison of charge sensitivity results of a single-ended (SE) accelerometer B\&K 8305-001 on two different vibration exciters: a PTB modified version of the Bouche shaker (VE) and a SPEKTRA SE-09 shaker. The results obtained exhibited a systematic deviation which increased with rising frequency, starting at $5 \mathrm{kHz}$. Relative deviations up to $2 \%$ at $10 \mathrm{kHz}$ and $4 \%$ at $20 \mathrm{kHz}$ were evidenced. It was also observed that instabilities of this order could have drawn the use of the SE artifact for the key comparison CCAUV.V-K2 into question.

According to this PTB study the cause of this deviation can be a material dependency of the measured magnitude of sensitivity. The VE and SE-09 shakers analyzed in their study use armatures made of beryllium and ceramics, respectively. Probable causes of this effect can be either differences in contact stiffness or modal deformation of the armature, where the acceleration input is interferometrically measured. This was justified by results of optical measurements on the base of a "naked" accelerometer (without case) and on the shaker armature close to the accelerometer. FEM analysis and scanning laser vibrometry measurements on the surface of the armatures have shown radial relative acceleration deviations. This proves that the armatures are not rigid bodies as they are normally assumed to be.

Bruns et al. have considered in a second publication [6] the inclusion of additional spring and damping elements in the mathematical modeling for a primary calibration of an SE accelerometer, in order to consider finite contact stiffness between the accelerometer and the armature. It was concluded that there are strong evidences that the cause of the problem is a non-negligible relative motion between the SE transducer and the shaker armature at high frequencies.

These studies carried out at PTB were restricted to calibration shakers models, which include lightweight armatures made of high stiffness materials. Most of these calibration exciters use air-borne bearings and present limited maximum force ratings. Other NMIs have different models of shakers in use, which can produce higher levels of deviations. These shakers are usually older models not initially designed for primary calibration purposes, which can be preferred in some specific conditions. For example, this might be the case when higher acceleration levels are required at high frequencies, when the fringe disappearance or minimum point methods are used [1].

The problem regarding low stiffness of the armature was already presented by the authors of this paper in a former publication [7]. It was shown that the charge sensitivity values obtained for a standard single-ended accelerometer mounted on three different vibration exciters presented large deviations above $1 \mathrm{kHz}$ due to modal deformations. The results obtained at $3 \mathrm{kHz}$ for two aluminium-armature shakers were much higher than the results obtained with an air-bearing guided beryllium-armature model. The authors have also introduced that using a high stiffness adapter it was possible to get equivalent results using aluminium and beryllium made armatures.

\section{MATHEMATICAL MODEL AND SIMULATIONS}

Piezoelectric accelerometers can be represented as a lightly damped single degree-of-freedom spring-mass system. In order to simplify the analysis, it will be considered here that the accelerometer is ideally mounted with infinite stiffness to a rigid body type armature. In this case, accelerometers can be modelled by the classical second order differential equation whose solution yields on the following frequency-dependent sensitivity equations

$$
\begin{aligned}
& S(f)=\frac{S_{0}}{\sqrt{\left(1-\left(\frac{f}{f_{n}}\right)^{2}\right)^{2}+4 \varsigma^{2}\left(\frac{f}{f_{n}}\right)^{2}}} \\
& \Delta \varphi(f)=-\arctan \left(\frac{2 \varsigma\left(\frac{f}{f_{n}}\right)}{1-\left(\frac{f}{f_{n}}\right)^{2}}\right)
\end{aligned}
$$

where $f_{n}$ is the undamped natural frequency in $\mathrm{Hz}, f$ is frequency in $\mathrm{Hz}, S(f)$ is the sensitivity magnitude at frequency $f, S_{0}$ is the low frequency reference sensitivity magnitude, $\Delta \varphi(f)$ is the sensitivity phase shift at frequency $f, \zeta$ is the damping factor.

If a high stiffness mechanical connection between the accelerometer and the armature is not achieved, a reduced mounted resonance frequency might be observed in the measured frequency response function. In this case, an abnormal increase of the accelerometer sensitivity occurs at high frequencies and significant effects can be seen above $5 \mathrm{kHz}$.

Most common accelerometers and reference grade accelerometers have resonances in the $20 \mathrm{kHz}$ to $60 \mathrm{kHz}$ range. Table 1 presents the nominal mounted resonance frequencies of single-ended reference accelerometers from different manufacturers. For practical use and for the simulations 
Table 1. Nominal mounted resonance frequencies of SE reference accelerometers obtained from their technical specifications.

\begin{tabular}{|l|c|c|c|}
\hline Manufacturer & model & Base material & Frequency \\
\hline Brüel \& Kjaer & $8305-001$ & $\begin{array}{c}316 \text { stainless } \\
\text { steel }\end{array}$ & $34 \mathrm{kHz}$ \\
\hline Endevco & $2270 \mathrm{M} 8$ & $\begin{array}{c}17-4 \text { PH stainless } \\
\text { steel }\end{array}$ & $55 \mathrm{kHz}$ \\
\hline Kistler & $8002 \mathrm{~K}$ & $\begin{array}{c}303 \text { stainless } \\
\text { steel }\end{array}$ & $40 \mathrm{kHz}$ \\
\hline
\end{tabular}

presented in sequence, $f_{n}$ will be approximated by the accelerometer mounted resonance frequency.

In order to evaluate the effect of the change in $f_{n}$ relative to the nominal value specified by the manufacturer, some simulations were performed using eq. (1) and (2). Measurements carried out at INMETRO have shown that the damping factor of an accelerometer model B\&K 8305-001 is approximately $\zeta=5 \times 10^{-3}$. This value will be used here for the simulations.

Figure 1 presents simulation results considering an accelerometer B\&K 8305-001. It shows the difference between sensitivity values obtained for different mounted resonance frequencies and the values relative to a nominal mounted resonance frequency of $34 \mathrm{kHz}$.

It can be seen that a change in the mounted resonance frequency of a SE accelerometer affects considerably its sensitivity response. If the mounted resonance frequency is reduced, an increase in the magnitude of the sensitivity and a phase shift reduction occur. This effect increases with the vibration frequency. For example, in the case of a mounted resonance frequency $5 \%$ lower than $34 \mathrm{kHz}$, the sensitivity magnitude is increased by $1 \%$ at $10 \mathrm{kHz}$ and $5.7 \%$ at $20 \mathrm{kHz}$. The influence on phase shift is reduced by approximately $0.01^{\circ}$ at $10 \mathrm{kHz}$ and $0.06^{\circ}$ at $20 \mathrm{kHz}$.

\section{MOUNTING SURFACE CONDITIONS}

As already mentioned, one important factor that affects the mounting stiffness of an accelerometer is the quality of the matting surfaces. It is important to assure that both the SE accelerometer base and the surface which it will be attached to are free of scratches and present proper flatness and roughness. The use of a proper lubricant as light oil or silicon grease helps to assure a high mounting stiffness and obtain the highest possible mounted resonance frequency.

During the torque application to stud mounted

Difference of sensitivity magnitude $x$ resonance frequency

(Reference condition $-\mathrm{fn}=\mathbf{3 4} \mathrm{kHz}$ )

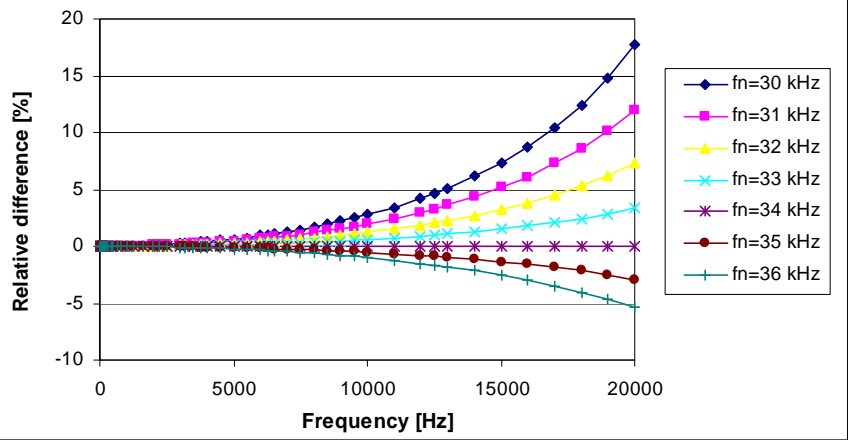

(a) accelerometers it is usual to appear circular scratches on its base. This might cause an increasing degradation of the mounting quality condition with time. One possible way to overcome this problem is to submit the accelerometer to relapping in order to recover its original condition.

Figure 2 shows the effect of the quality of mounting base on the sensitivity of a SE accelerometer measured by a primary laser interferometric system at INMETRO. This figure includes both the measurement results obtained with the accelerometer as received from a customer and after a re-lapping of its base was performed. The increase of the mounted resonance after improvement of the mounting surface condition is clearly noted in frequencies above $5 \mathrm{kHz}$. The relative difference on the measured sensitivity in this case was $-1.1 \%$ at $10 \mathrm{kHz}$ and $4.5 \%$ at $20 \mathrm{kHz}$.

During a typical vibration interlaboratory key comparison [3] it is usual to have more than ten participating laboratories performing around five repeated series of calibrations. Considering that each series includes two mountings to minimize the coupling effect between transverse accelerations generated by the vibration exciter and the transverse sensitivity of the accelerometer, this would require a minimum of fifty mountings. If in addition ten preliminary measurements are carried out by the pilot laboratory to access the stability of the artifact and another ten monitoring measurements are carried out along the circulation period, it is easy to reach a number over one hundred mountings. This high number of mountings might require several re-lappings of the accelerometer base during the period of the comparison in order to assure that every participant receives the DUT with approximately the same mechanical conditions.

However, the re-lapping process requires special technical skills; it might pose risks to the integrity of the artifact and is based on the removal of material. If several re-lappings are necessary, some change in the sensitivity can be expected.

If the number of mountings is reduced considerably, the relapping process can be eliminated and the accelerometer would probably maintain its original characteristics with minimum risks.

\section{PROPOSAL OF ADAPTER}

A very simple solution has been used at INMETRO to reduce the number of mountings and the dispersion between the results obtained using different vibration exciters. The accelerometer is stud mounted onto the top surface of a

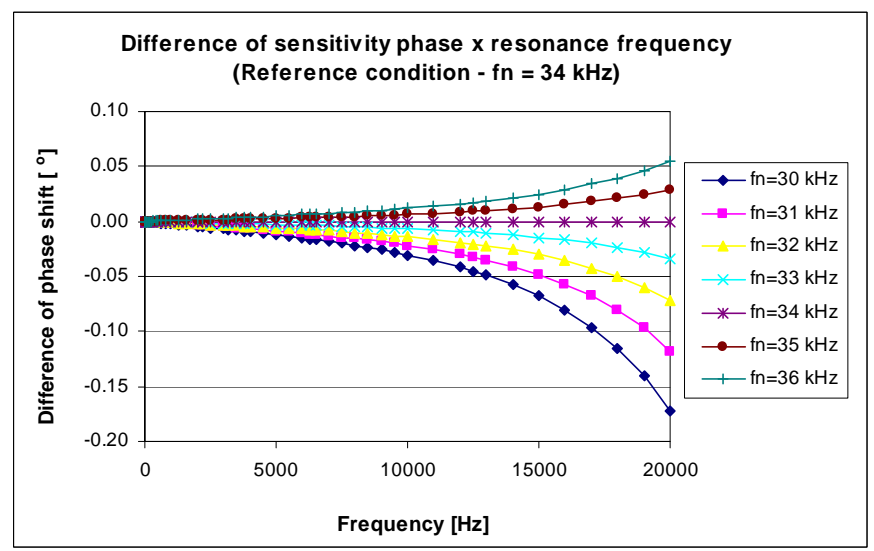

(b)

Figure 1. Difference of the sensitivity (a) magnitude and (b) phase of a SE accelerometer from a reference mounted resonance frequency of $34 \mathrm{kHz}$ 


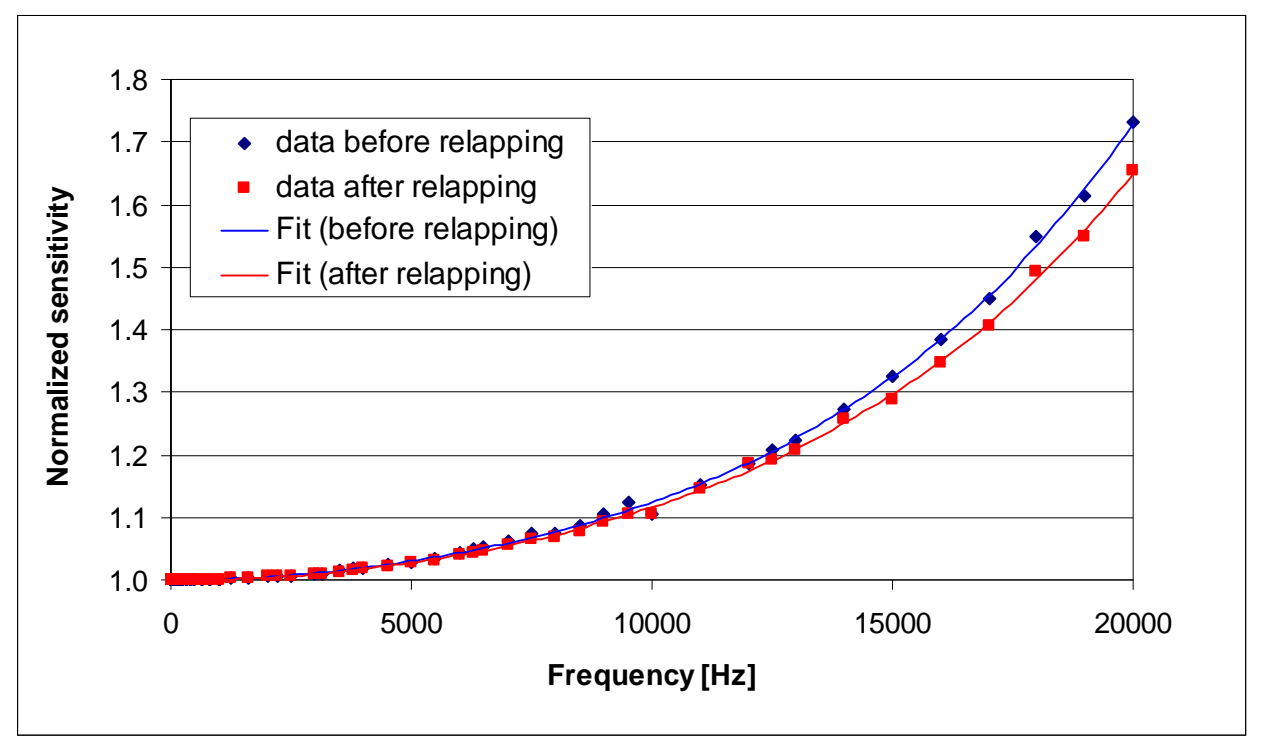

Figure 2. Normalized sensitivity magnitude measured before and after relapping the base of a SE accelerometer.

mechanical adapter, which is then fixed to the armature of the different shakers available in our vibration laboratory. This adapter can be attached directly to the shaker armature or via a secondary adapter to fit it to the different fixture patterns. These additional adapters can also be used to allow rotation of the set at specific angles.

Figure 3 shows the mechanical design of an ANSI 304 stainless steel adapter. Its top surface is polished for direct optical reflection of the laser beam. It includes a \# 10-32 UNF threaded through hole for stud mounting of the accelerometer and a transverse $3 \mathrm{~mm}$ diameter hole to help providing the required mounting torque. The $17 \mathrm{~mm}$ diameter neck is to reduce mass and allow the mounting on the armature of a B\&K calibration head type 4815 .

Figure 4 shows a single-ended reference accelerometer B\&K 8305-001 mounted on the top surface of this stainless steel adaptor. The red lines illustrate laser beams reflecting onto its polished top reference surface.

This adapter allows direct mounting to armatures of many different vibration exciters. This is exemplified in Figure 5, which shows the accelerometer plus adapter set mounted on the following models: (a) B\&K high $g$ shaker head model 4811; (b) B\&K shaker model 4809; (c) Endevco model 2901; and (d) PCB shaker model 396C10.

An additional adapter might be necessary to mount the system shown in Figure 4 to some exciters like the B\&K calibration head model 4815, which presents a recessed mounting plane. Figure 6 (a) presents a square shaped

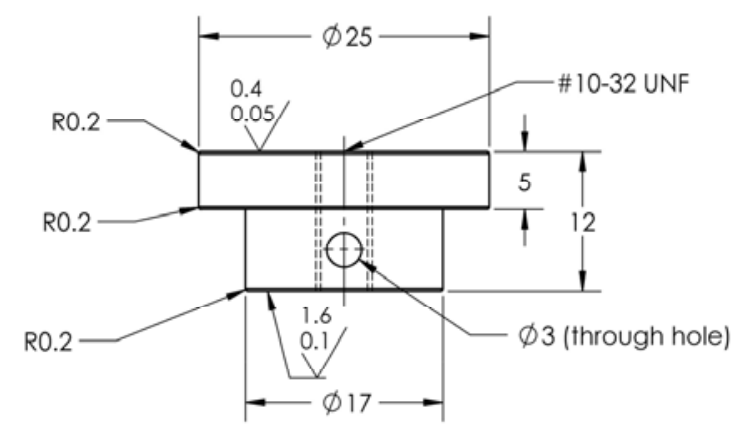

Figure 3. Mechanical design of the mounting adapter for SE accelerometers. aluminium adapter to allow proper fitting to the mounting hole pattern of this shaker and to raise its mounting plane. This aluminium adapter also allows rotation of the set in $90^{\circ}$ angle increments. The final mounting of the set onto the shaker armature is shown in Figure 6 (b).

It should be noted that despite the lower stiffness of this inseries aluminium adapter, it has negligible influence on the calibration results. The original shaker armature and adapter provided by $B \& K$ are also made of the same material and its

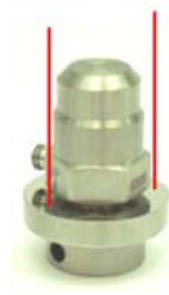

Figure 4. SE reference accelerometer mounted on top of the stainless steel mounting adapter.

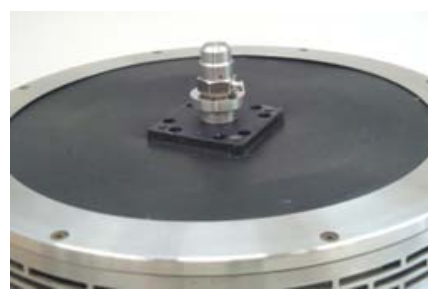

(a)

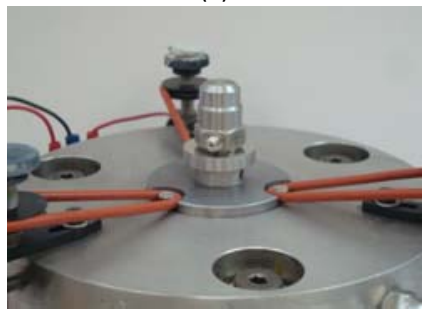

(c)

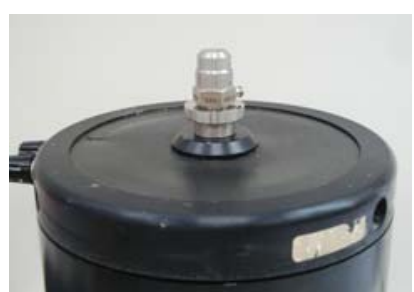

(b)

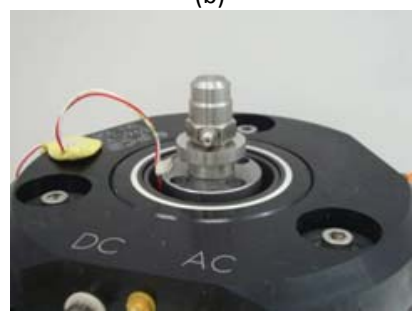

(d)
Figure 5. SE reference accelerometer and adapter mounted on top of the moving element of different vibration exciters: (a) BK $4805+$ BK 4811 high $g$ head; (b) BK 4809; (c) Endevco 2901; (d) PCB 396C10. 


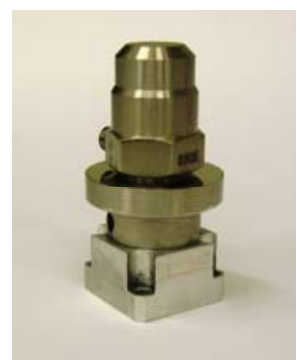

(a)

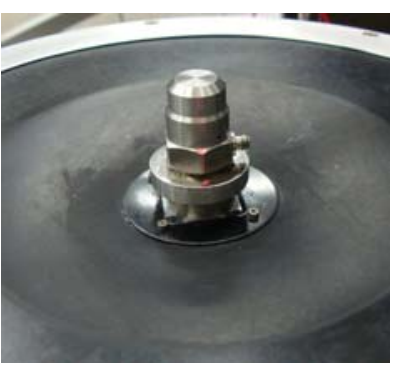

(b)
Figure 6. (a) SE reference accelerometer and stainless steel adapter mounted on an additional aluminium adapter and this set (b) mounted on the moving element of a vibration exciter BK $4805+$ BK 4815 calibration head.

low weight helps to reduce the total mass loading mounted on the top of exciter.

Finite elements modal analyses have shown that the proposed adapter presents resonance frequencies far beyond the frequency range of interest. The two lower resonance frequencies refer to tilting modes near $30 \mathrm{kHz}$ and to a rotational mode at $32 \mathrm{kHz}$, approximately. These modes are shown in Figure 7 (a), (b) and (c) respectively. The first flexural mode of the top reference surface, which occurs above $58 \mathrm{kHz}$ is shown in Figure 7 (d). This flexural deflection mode would very probably introduce errors on the acceleration determined by primary laser interferometric methods and could additionally induce stress in the piezoelectric discs inside the accelerometer under test.

\section{MAINTENANCE OF A FIXED REFERENCE CONDITION}

Figure 8 illustrates how (a) modal deformation of the shaker armature and (b) finite mounting stiffness can generate errors to the calibration of accelerometers by laser interferometric measurements. Changes in the distance between the accelerometer reference plane (i.e. the $\mathrm{SE}$ accelerometer base) and the laser measurement plane (i.e. the armature surface) can cause large systematic errors to the determination of the accelerometer sensitivity.

The use of the adapter proposed by the authors helps to maintain a fixed measurement condition, with very good independence to the shaker used. The distance between the accelerometer reference plane and the interferometric

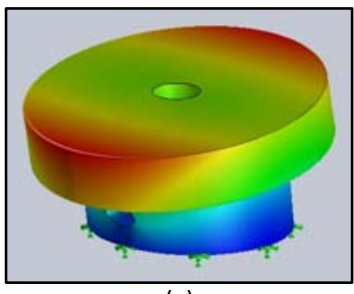

(a)

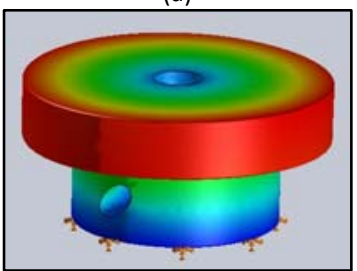

(c)

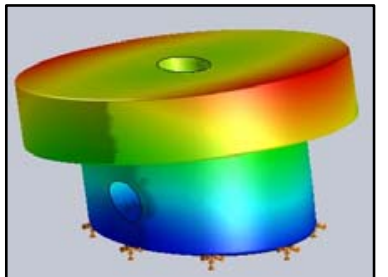

(b)

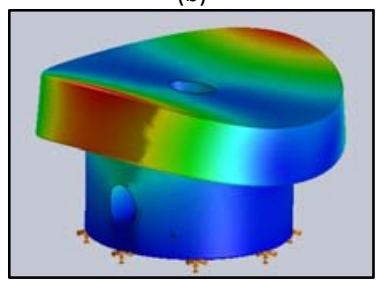

(d)
Figure 7. First four vibrational modes of the adapter: (a) mode 1 - tilt mode at $29657 \mathrm{~Hz}$; (b) mode 2 - tilt mode at $30032 \mathrm{~Hz}$; (c) mode 3 -torsional mode at $32198 \mathrm{~Hz}$; (d) mode $4-$ flexural mode at $58317 \mathrm{~Hz}$. measurement plane is kept constant even if the armature presents flexural modes within the frequency range of measurement and a poor mounting stiffness is obtained between the adapter and the shaker armature. These two conditions are exemplified in Figure 9 (a) and (b), respectively.

\section{MEASUREMENT RESULTS}

In order to exemplify the application of the proposed adapter, and to illustrate the level of improvement achieved, some experimental results are presented in this section.

\subsection{Measurements on an air bearing shaker}

The frequency response of a SE standard accelerometer B\&K 8305-001 mounted with the stainless steel adapter on the beryllium moving element of a vibration exciter Endevco 2901 is shown in Figure 10. The acceleration measurements were performed at two diametrically opposed points (M1P1 and M1P2) on the surface of the adapter by a laser vibrometer Polytec model VDD-660. The calculated mean sensitivity curve (Mean M1) presents a peak value at approximately $33.5 \mathrm{kHz}$, which is only $1.47 \%$ lower than the nominal mounted resonance frequency given in the technical specifications of the accelerometer.

This level of agreement provides an evidence of the good quality of the mounting stiffness. The knowledge of the mounted resonance frequency allows the calculation of the magnitude and phase responses of an accelerometer using equations (1) and (2) respectively for comparison with the experimentally determined sensitivities.

Even with the use of the adapter, the presence of some glitches on the frequency response functions obtained was observed. The cause of these glitches was investigated and apparently they are motivated by internal resonances of the accelerometer.

In order to verify the feasibility of this assumption, the authors have carried out some finite element analyses on a simplified model of the accelerometer under test. The first six vibration modes obtained from this analysis are presented in Figure 11.

Figure 11 shows the presence of five vibrational modes before the first axial longitudinal resonance. Two tilting modes of the seismic element occur around $7.6 \mathrm{kHz}$, while one rotational mode shows up at $15.5 \mathrm{kHz}$. Flexural and longitudinal case modes appear close do $24 \mathrm{kHz}$. The main longitudinal mode obtained with our simplified model was

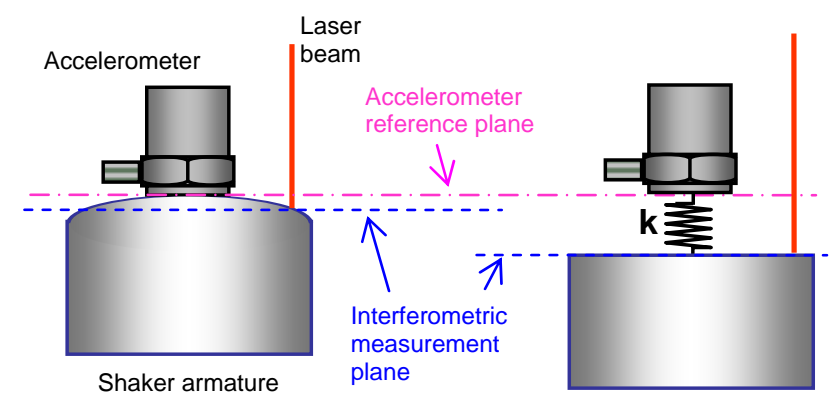

(a)

(b)

Figure 8. Mismatch between the SE reference accelerometer plane and the interferometric measurement plane due to (a) modal deflections on the shaker armature and (b) poor mounting stiffness. 


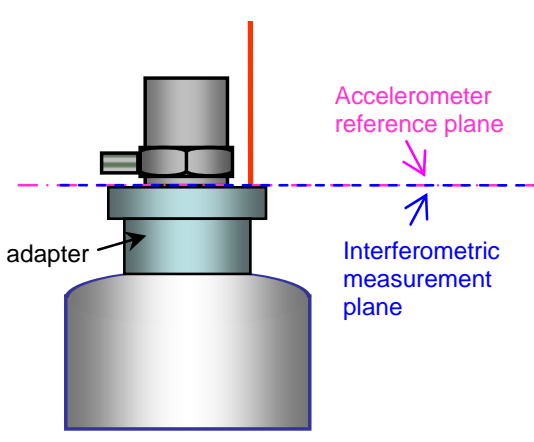

(a)

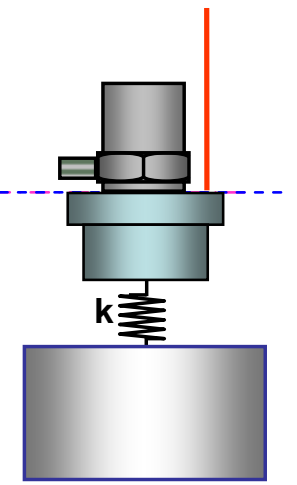

(b)
Figure 9. Maintenance of a fixed reference condition between the SE accelerometer and the interferometric measurement plane with the use of an adapter even in case of (a) modal deflections on the shaker armature and (b) poor mounting stiffness between the adapter and shaker armature.

observed close to $34 \mathrm{kHz}$.

Given the good match between the frequencies of the glitches on the measured FRF and the resonance frequencies obtained by the FE analysis, it is clear to the authors that the remaining causes of errors are due to internal resonances in the accelerometer. Figure 12 also shows that the averaging of the results measured at different points improve the final FRF obtained. The major effect of the rotational mode can be explained by the characteristics of the shaker used for these measurements. Since the armature is guided by air-bearings and is suspended by rubber O-rings, it does not offer strong mechanical constrains to rotations. This might be the reason that rotational modes cause larger glitches on the FRF than the tilting and the flexural case resonances.

\subsection{Comparison between results obtained in different shakers}

Figure 13 presents a comparison between the results of charge sensitivity measured using a flat-spring-suspension exciter model B\&K 4815 against the results obtained using an air-bearing guided beryllium-armature, exciter model PCB 396C10. The sensitivities presented are normalized by the reference value measured at $160 \mathrm{~Hz}$. These measurements were carried out with the $\mathrm{B} \& \mathrm{~K}$ exciter using the adapters shown in Figure 6(a) and with the PCB shaker with and also without the stainless steel adapter.

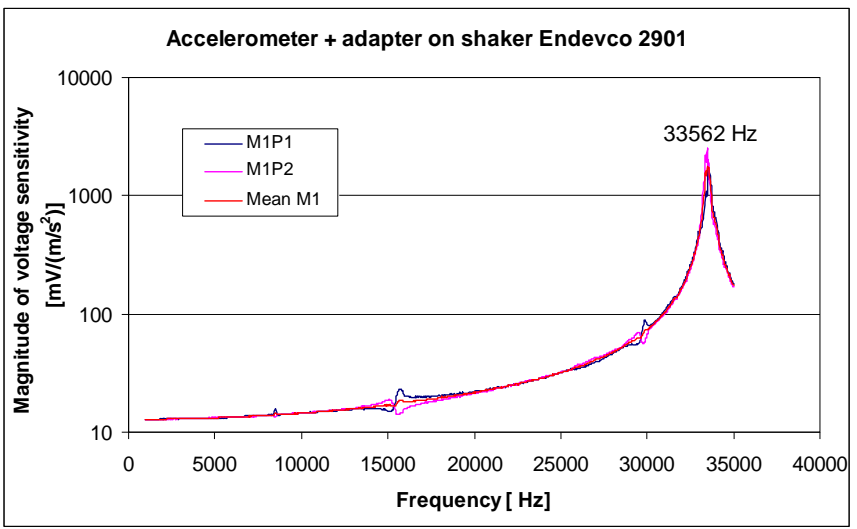

Figure 10. Magnitude of the FRF of an accelerometer B\&K 8305-001+ adapter mounted on the moving element of a vibration exciter Endevco 2901.
The results obtained using the accelerometer mounted directly onto the beryllium armature of the PCB shaker present a systematic increase of the sensitivity at high frequencies. Deviations of $0.9 \%$ and $3.5 \%$ are observed at $10 \mathrm{kHz}$ and $20 \mathrm{kHz}$, respectively. Using the stainless steel adapter on both shakers, a very good agreement is obtained. The deviations at these same frequencies are then reduced to $0.04 \%$ and $0.3 \%$. It should be noted that the residual effect on the sensitivity seen at $17 \mathrm{kHz}$ is caused by an internal resonance of the accelerometer. Apart from this frequency, very smooth curves
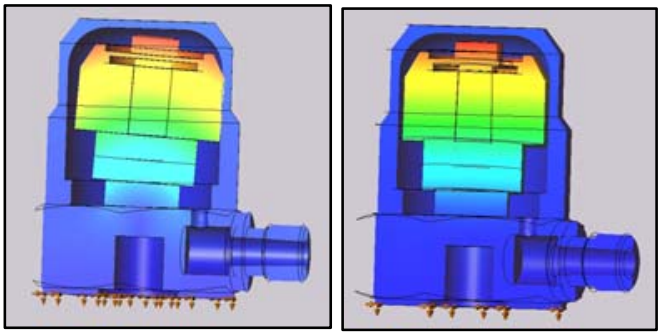

(a) Modes 1 and 2 - Seismic mass tilting modes, $7575 \mathrm{~Hz}$ and $7682 \mathrm{~Hz}$

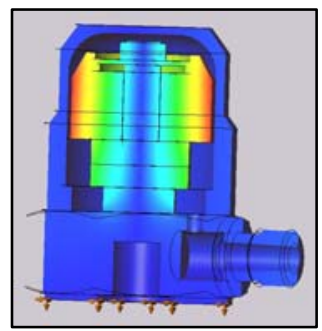

(b) Mode 3 - Seismic mass rotational mode, $15570 \mathrm{~Hz}$

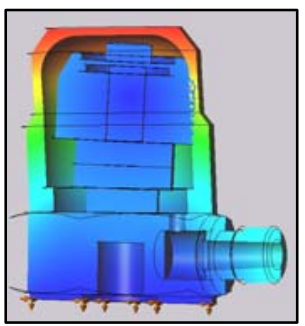

(c) Mode 4 - Case flexural mode, $24514 \mathrm{~Hz}$

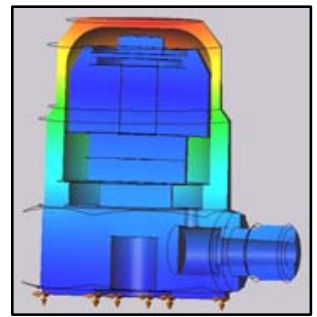

(d) Mode 5 - Case longitudinal mode, $24682 \mathrm{~Hz}$

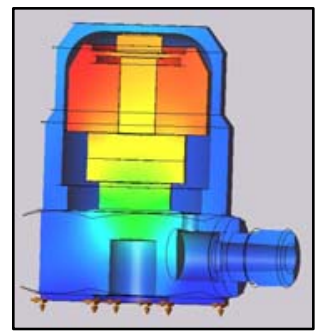

(e) Mode 6 - Sensor longitudinal mode, $34127 \mathrm{~Hz}$

Figure 11. Vibrational modes obtained by FEA applied to a simplified model of an accelerometer B\&K 8305-001. 


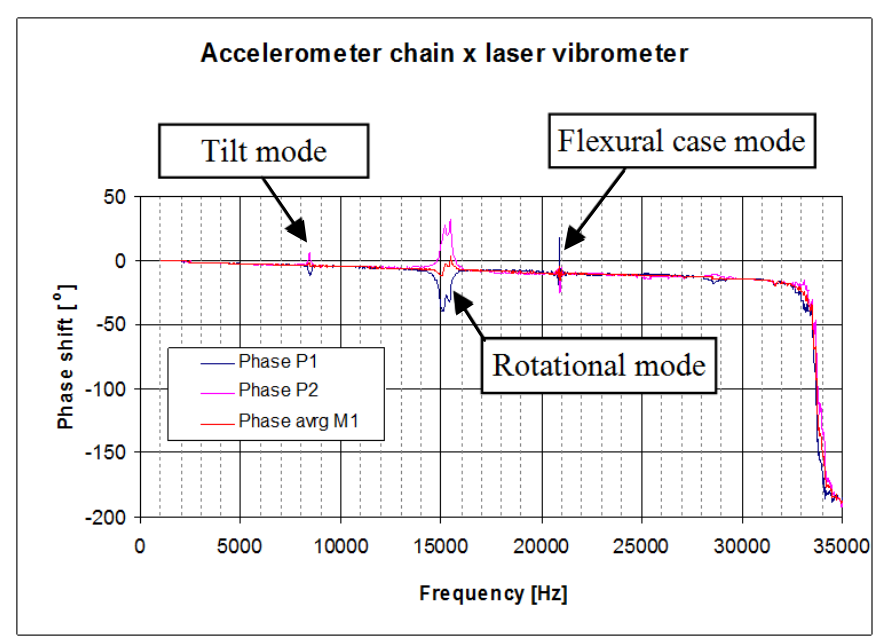

Figure 12. Phase of the FRF of an accelerometer B\&K 8305-001 + adapter mounted on the moving element of a vibration exciter Endevco 2901.

are obtained.

A similar behaviour has also been observed when using a vibration exciter Endevco model 2901 both with and without the stainless steel adapter. This other shaker also includes an air bearing guided beryllium-armature.

The reduction in the dispersion at high frequencies is evidenced in Figure 14, which presents the relative difference between sensitivity magnitude results obtained using three different shakers with and without the adapter proposed. The relative values are calculated in reference to the sensitivity results obtained employing a shaker B\&K 4815 with the mounting configuration shown in Figure 6. In order to facilitate visualization of the improvement achieved, this graph includes polynomial curves fitted to the measured data and excludes some few spurious frequencies contaminated by internal resonances of the accelerometer. The agreement between the sensitivity magnitude results obtained using the adapter on these three different shakers is within $\pm 0.3 \%$ between $1 \mathrm{kHz}$ and $10 \mathrm{kHz}$, excluding $5.5 \mathrm{kHz}$ where a deviation of $0.4 \%$ is observed.

\section{CONCLUSIONS}

A study of the dispersion on primary calibration results of SE accelerometers has been presented in this paper. It was confirmed that the results obtained at high frequencies can differ significantly depending on the vibration exciter used. This effect might have different causes, including the different mounting stiffness conditions and modal deformations on the armature of vibration exciters.

Considering the fact that SE transfer accelerometers are intended to calibrate BTB standards or calibration shakers with built-in reference accelerometers, it is important to calibrate them in the same condition or as close as possible to its end use.

Currently, exciters with armatures made of aluminium, beryllium, and ceramics are available at NMIs for use in calibration of accelerometers. This variability of mounting conditions might yield in different mounted resonance frequencies, which then affects the sensitivity results obtained by each laboratory at high frequencies.

This paper focuses on a proposal that can minimize the dispersion problem. It was shown that the use of a stainless steel mounting adapter with high contact stiffness properties and no vibration modes within the frequency range of interest can improve considerably the agreement between the results obtained. Some experimental results have been presented to demonstrate that substantial improvement is obtained and the dispersion can be significantly reduced. This reduction of the effect of the vibration exciter can help laboratories to obtain better comparability between different calibration systems. This is an important issue during the stage of validation of different calibration systems or even comparison between different calibration methods.

An important impact of the use of an adapter can be related to primary interlaboratory comparisons. If future comparisons consider this proposal, the dispersion caused by variation of the mounting stiffness due to the use of different exciters by the participants can be minimized. In consequence, a better agreement might be achieved and then a comparison reference value with lower uncertainty can be obtained.

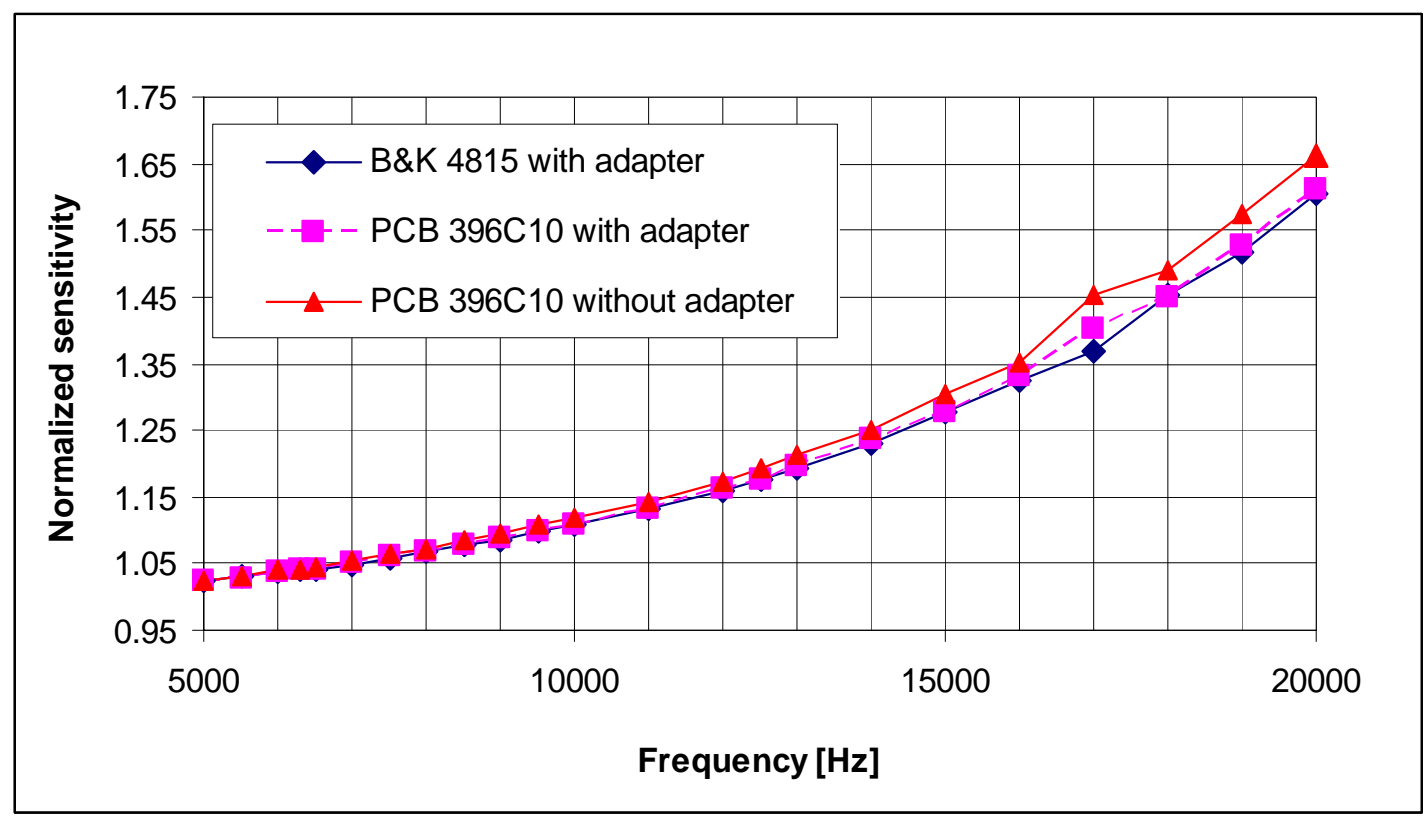

Figure 13. Normalized sensitivity results obtained using an exciter BK 4815 with adapter and an exciter PCB $396 C 10$ with and without adapter. 


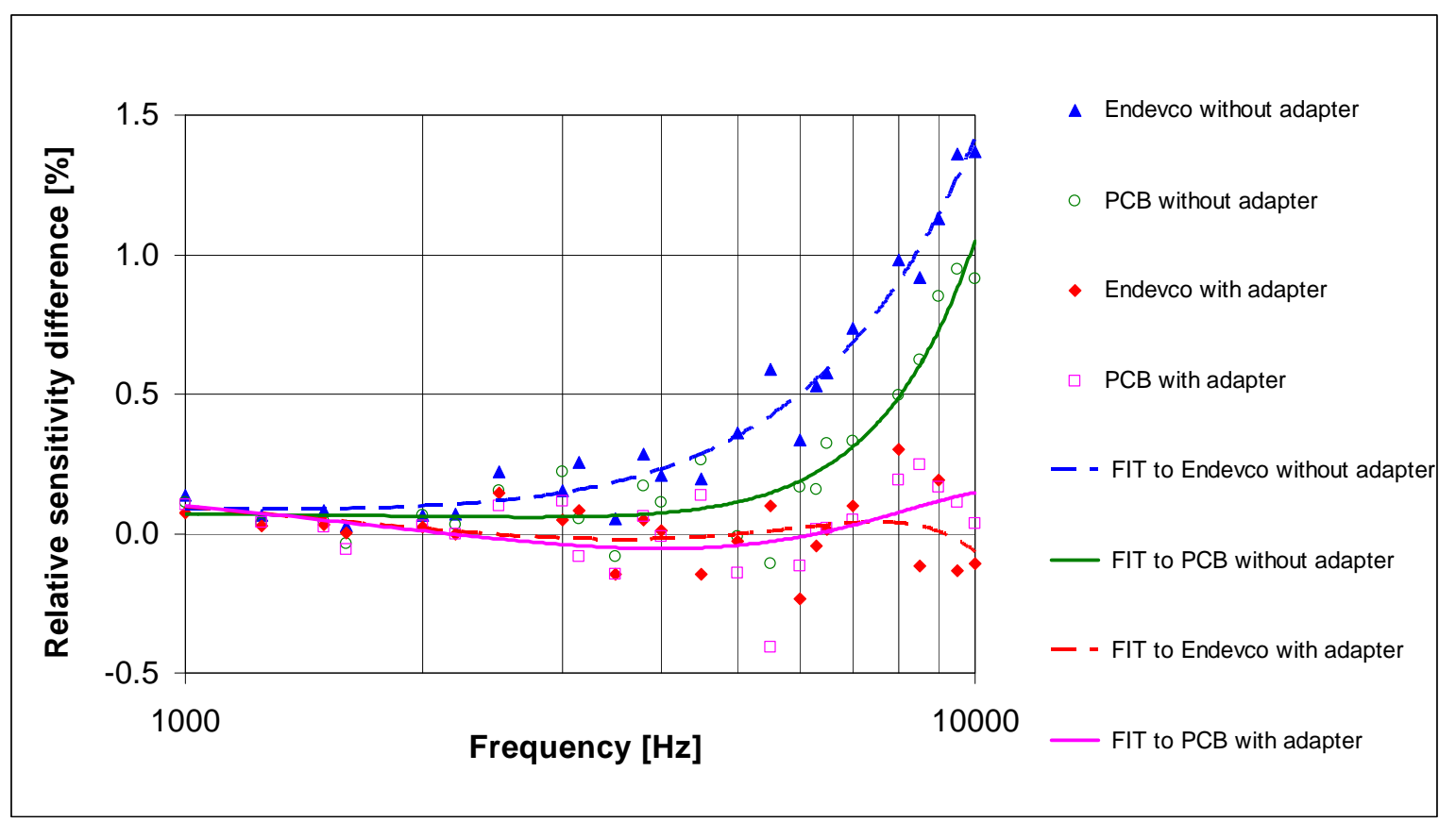

Figure 14. Difference of sensitivity results using exciters Endevco 2901 and PCB $396 C 10$ relative to the ones obtained using a B\&K 4815 with adapter.

Considering this, the authors are proposing the inclusion of an adapter together with the accelerometers to be circulated for the future regional key comparison SIM.AUV.V-K2.

The use of a similar adapter by different laboratories could provide a better comparability between their calibration capabilities. Additionally, comparing results with and without the common adapter, would be possible to determine correction factors between the results obtained with different vibration exciters.

\section{REFERENCES}

[1] ISO, International Standard 16063-11, Methods for the calibration of vibration and shock transducers - Part 11: primary vibration calibration by laser interferometry, International Organization for Standardization, Geneva, 1999.

[2] BIPM, Measurement comparisons in the context of the CIPM MRA, CIPM MRA-D-05 version 1.3, October 2012.

[3] H.J. von Martens, C. Elster, A. Link, A. Täubner, W. Wabinski, Final report on key-comparison CCAUV.V-K1, Metrologia vol.
40, tech. suppl. 09001, 2003 doi:10.1088/00261394/40/1A/09001.

http://www.bipm.org/utils/common/pdf/final_reports/AUV/ V-K1/CCAUV.V-K1.pdf.

[4] T. Bruns, Technical protocol of the CIPM key comparison CCAUV.V-K2, 2009,

http://kcdb.bipm.org/appendixB/appbresults/CCAUV.V-

K2/CCAUV.V-K2_Technical_Protocol.pdf.

[5] A. Täubner, H. Schlaak, M. Brucke, T. Bruns, The influence of different vibration exciter systems on high frequency primary calibration of single-ended accelerometers, Metrologia vol. 47, No.1, 2010, pp.58-64, doi:10.1088/0026-394/47/1/007.

[6] T. Bruns, A. Link, A. Täubner, The influence of different vibration exciter systems on high frequency primary calibration of single-ended accelerometers: II, Metrologia vol. 49, No.1, 2012, pp.27-31, doi:10.1088/0026-1394/49/1/005.

[7] G.P. Ripper, R.S. Dias, G.A. Garcia, Primary accelerometer calibration problems due to vibration exciters, Measurement vol. 42, No.9, 2009, pp. 1363-1369,

doi:10.1016/j.measurement.2009.05.002. 